\title{
The Capodimonte Deep Field ${ }^{\star}$
}

\section{Presentation of the survey and first follow-up studies $\star \star$}

\author{
J. M. Alcalá ${ }^{1}$, M. Pannella ${ }^{2,1}$, E. Puddu ${ }^{1}$, M. Radovich ${ }^{1}$, R. Silvotti ${ }^{1}$, M. Arnaboldi ${ }^{3}$, M. Capaccioli ${ }^{1,4}$, G. Covone ${ }^{8,1}$,

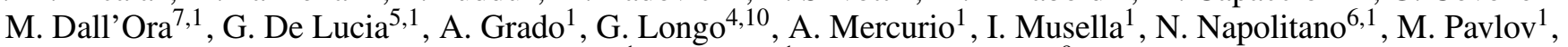 \\ A. Rifatto ${ }^{1}$, V. Ripepi ${ }^{1}$, and R. Scaramella ${ }^{9}$
}

\author{
1 INAF - Osservatorio Astronomico di Capodimonte, via Moiariello 16, 80131 Napoli, Italy \\ e-mail: jmae@na.astro.it \\ 2 Max - Planck Institut für Extraterrestrische Physik, Giessenbachstrasse, 85748 Garching bei München, Germany \\ 3 INAF - Osservatorio Astronomico di Torino, via Osservatorio 2010025 Pino Torinese, Italy \\ 4 Dipartimento di Scienze Fisiche, Università Federico II, Napoli, Italy \\ 5 Max Planck Institut für Astrophysik, 85748 Garching bei München, Germany \\ ${ }^{6}$ Kapteyn Astronomical Institute, Landleven 12, 9747 AD Groningen, The Netherlands \\ 7 Dipartimento di Fisica, Universita' di Roma Tor Vergata, via della Ricerca Scientifica 1, 00133 Roma, Italy \\ ${ }^{8}$ Laboratoire d'Astrophysique de Marseille, BP 8, 13376 Marseille Cedex 12, France \\ 9 INAF - Osservatorio Astronomico di Monteporzio, via di Frascati 33, 00044 Roma, Italy \\ ${ }^{10}$ INFN - Napoli Unit, via Cinthia 6, 80126 Napoli, Italy
}

Received 22 July 2003 / Accepted 5 August 2004

\begin{abstract}
We present the Capodimonte Deep Field (OACDF), a deep field covering an area of $0.5 \mathrm{deg}^{2}$ in the $B, V, R$ optical bands plus six medium-band filters in the wavelength range 773-913 $\mathrm{nm}$. The field reaches the following limiting magnitudes: $B_{A B} \sim 25.3, V_{A B} \sim 24.8$ and $R_{A B} \sim 25.1$ and contains $\sim 50000$ extended sources in the magnitude range $18 \leq R_{A B} \leq 25.0$. Hence, it is intermediate between deep pencil beam surveys and very wide but shallow surveys. The main scientific goal of the OACDF is the identification and characterization of early-type field galaxies at different look-back times in order to study different scenarios of galaxy formation. Parallel goals include the search for groups and clusters of galaxies and the search for rare and peculiar objects (gravitational lenses, QSOs, halo White Dwarfs). In this paper we describe the OACDF data reduction, the methods adopted for the extraction of the photometric catalogs, the photometric calibration and the quality assessment of the catalogs by means of galaxy number counts, spectroscopic and photometric redshifts and star colors. We also present the first results of the search for galaxy overdensities. The depth of the OACDF and its relatively large spatial coverage with respect to pencil beam surveys make it a good tool for further studies of galaxy formation and evolution in the redshift range $0-1$, as well as for stellar studies.
\end{abstract}

Key words. surveys - catalogs - galaxies: clusters: general

\section{Introduction}

Wide and deep surveys are a vital tool in modern astronomy: they provide the large datasets of objects needed (both at high and low redshifts) to improve our knowledge of the physical mechanisms driving galaxy formation and evolution.

\footnotetext{
* Based on observations carried out at the European Southern Observatory, La Silla, Chile under proposals numbers 63.O-0464(A), 64.O-0304(A), 65.O-0298(A) and 67.B-0457(A).

$\star \star$ Tables A.1-A.3 are only available in electronic form at the CDS via anonymous ftp to

cdsarc.u-strasbg.fr $(130.79 .128 .5)$ or via

http://cdsweb.u-strasbg.fr/cgi-bin/qcat?J/A+A/428/339
}

Recent wide field surveys, such as DPOSS, 2MASS, SDSS and 2DF, (Djorgovski et al. 2000; Colless et al. 2001; Jarrett et al. 2000; York et al. 2000) provide an unprecedented wealth of information on the structure of the local Universe.

On the other hand, the complementary pencil beam deep surveys (i.e. relatively small patches of the sky observed in several bands with high angular resolution and high $S / N$ ratios at faint magnitude levels) allow us to investigate the high- $z$ Universe $(z \gtrsim 1)$, providing large sets of distant galaxies to constrain the different evolution scenarios.

In the past, the significance of deep fields was limited by the constraints of the small field of view covered by single CCD detectors and the need to obtain large fractions of observing time with $4 \mathrm{~m}$ class telescopes. The small spatial coverage of 
the first studies (Tyson 1988; Lilly et al. 1995; Ellis et al. 1996; Le Fevre et al. 1996) succeeded in tracing only a rough and broad picture of galaxy evolution in the redshift range $0<z<$ 0.8 and therefore did not allow strong constraints on the various scenarios proposed for galaxy formation and evolution (White \& Frenk 1991; Cole et al. 2000; Aragon-Salamanca et al. 1998; Peebles 2002).

The Hubble Deep Fields (e.g. Ferguson et al. 2000) has marked a cornerstone in our understanding of galaxy evolutionary processes, providing unprecedented contraints on the theoretical scenarios for galaxy formation and evolution.

Similar deep surveys have been recently performed using ground-based telescopes of the $10 \mathrm{~m}$ class generation: the Subaru Deep Field (Maihara et al. 2001), the Fors Deep Survey (Heidt et al. 2003) and the Gemini Deep Deep Survey (Abraham et al. 2004). However, an important drawback of these deep surveys is that the typical field of view has angular sizes at $z \sim 1$ which are relatively small with respect to the scales relevant for large-scale structure. Thus, in order to study the properties of distant galaxies at high redshift in different large scale environments, it is mandatory to control field-tofield variations, by means of deep surveys along many different lines of sight or extended to larger contiguous sky areas.

The advent of wide field imagers on dedicated survey telescopes, like the wide-field imager (WFI) at the ESO/MPG 2.2 m telescope, allow one to perform deep surveys on sky areas of the order of 1 square degree, thus providing an ideal tool to gather high accuracy data for statistically significant samples of objects in the redshift range $0<z<1$. This has prompted the observation of several deep fields at optical and near infrared wavelengths: COMBO-17 (Wolf et al. 2003); K20 (Cimatti et al. 2002), MUNICS (Drory et al. 2001), the VIRMOS-VLT deep field (Le Fevre et al. 2003; Mc Cracken et al. 2003; Radovich et al. 2004).

While these surveys cannot, in general, compete with the HDFS and the $10 \mathrm{~m}$ class telescope deep surveys in terms of depth, they allow one to study in detail the galaxy population up to $z \simeq 1$ in a much larger field of view.

This fact is extremely important, for instance, when determining the luminosity function and its possible evolution up to $z \simeq 1$ (e.g., Wolf et al. 2003).

In 1999, the Osservatorio Astronomico di Capodimonte (INAF-OAC) started the Capodimonte Deep Field (OACDF) project using WFI at the ESO/MPG $2.2 \mathrm{~m}$ telescope at La Silla, Chile. The OACDF covers an area of $0.5 \mathrm{deg}^{2}$ in the $B, V, R$ optical bands and in six medium-band filters in the wavelength range 773-913 $\mathrm{nm}$. The OACDF is intermediate between deep pencil beam surveys and very wide, but shallow surveys. As it will be shown in the following paragraphs the OACDF reaches limiting magnitudes $B_{A B} \sim 25.3, V_{A B} \sim 24.8$ and $R_{A B} \sim 25.1$ and contains $\approx 50000$ extended sources in the magnitude range $18 \leq R_{A B} \leq 25.0$.

The OACDF was complemented with a first spectroscopic follow-up and with a shallow photometric survey overlapping the deep survey and covering $1 \mathrm{deg}^{2}$ in the $B, V, R$ and $I$ bands. The shallow survey reaches a depth of $I_{A B} \sim 22.1$ and was planned in order to enhance the statistics at low redshift.
The OACDF observing strategy was driven mainly by the identification and characterization of a statistically significant sample of early-type galaxies at different redshifts and in different environments. The number density, as well as the global morphological and photometric properties of these objects, are predicted to vary for different formation scenarios (Aragon Salamanca et al. 1998; Kauffmann \& Charlot 1998; Somerville et al. 2004). The filter set-up and in particular the availabilty of medium band filters in the range 753-913 nm, allow us to trace the D4000 break over the redshift range from 0 to 1 and therefore to obtain an accurate estimate of the photometric redshifts for these objects.

\section{Parallel goals of the OACDF project are:}

- the search for groups and clusters of galaxies to study the luminosity function of galaxy clusters, as well as their luminosity and color segregation, both linked to the more general question of the evolution of galaxies in environments with different densities (Menanteau et al. 1999). Another aspect is to study the cluster morphology (clustering and sub-clustering) which bear relevant information on the history of the Universe (Navarro et al. 1996). The identification of a sample of groups of galaxies and intermediaterichness clusters of galaxies at intermediate redshift, extracted in a homogeneous way, is necessary to study the properties of galaxies in moderately dense environments;

- the search for rare/peculiar objects, in particular gravitational lenses and high-redshift QSOs $(z>3)$;

- the identification of nearby galaxies with strong emission lines using the medium-band filters to perform clustering and correlation function studies;

- to provide a photometric and astrometric database for stellar studies.

While some peculiar objects present in the OACDF have already been discussed elsewhere (cf. gravitational lenses in Sazhin et al. 2003; white dwarfs in Silvotti et al. 2003), in this paper we present the OACDF photometric calibration, the catalog extraction, the quality assessment of the photometric catalogs through the derivation of galaxy number counts, spectroscopy of stars and galaxies by means of photometric and spectroscopic redshifts. We also present the first results of the search for galaxy overdensities.

In a forthcoming paper, we will use the OACDF data, the photometric redshift determinations and the available spectra in order to analyze the redshift and environmental evolution of scaling-laws, such as the Kormendy relation (Kormendy 1977; Ziegler et al. 1999; La Barbera et al. 2003), and of the stellar populations of these objects through the use of the Lick indices (Faber et al. 1985; Worthey et al. 1992) and stellar population synthesis models (Thomas et al. 2003).

Other future OACDF papers will include the following tasks:

- search for groups and clusters of galaxies: in the present paper we describe the adopted methods for the detection of groups and clusters of galaxies in the OACDF (see Sect. 6). In a future paper we will use the candidate clusters as part 
of a larger statistical sample needed to derive the multiplicity function and to study the luminosity function as a function of the environment. Follow-up spectroscopic studies are in course;

- search for Gravitational lenses: an important by-product of a survey like the OACDF is the possible detection of strong gravitational lenses (GLs). We have therefore started an extensive search for GLs in the OACDF2 and OACDF4 fields by spectroscopic observations at the ESO $3.6 \mathrm{~m}$ telescope at La Silla, Chile. In a future paper we will present the methodology and the results of this search and, in particular, its implications for wider surveys with VST, which will allow us to discover several tens of bright GLs in the Southern emisphere;

- the spectroscopic confirmation of white dwarf (WD) candidates selected through their colors. Spectroscopic data were obtained at the ESO NTT and $3.6 \mathrm{~m}$ telescopes and are under reduction; some preliminary results are shown in Silvotti et al. (2003). The main goal of this project is to increase the WD statistics, in particular for the cooler, fainter and older objects, which contain crucial information on the genesis of our Galaxy: age of the galactic disk (and halo) through the WD luminosity function, initial mass function, and stellar formation rate (see Fontaine et al. 2001 for an updated review). Of particular interest is the halo WD population as these rare and faint objects, whose statistics are almost totally unknown, might partially contribute to the galactic dark matter. This is suggested by the results of the MACHO+EROS microlensing experiments which show that microlensing events are mainly produced by halo objects with an average mass of $\sim 0.5 M_{\odot}$ (Alcock et al. 2000).

The present paper is structured as follows: in Sect. 2 we present the field selection and the observational strategy for the imaging and initial spectroscopic follow-up. The data reduction and photometric calibration, as well as the systematic behavior of the zero points of the ESO-WFI CCDs, and the spectroscopic data reduction are reported in Sect. 3. In Sect. 4 we discuss the problems posed by catalog extraction, while the quality assessment of the OACDF catalogs are discussed in Sect. 5. First results on the search for groups and clusters of galaxies are reported in Sect. 6 and a summary is presented in Sect. 7. Finally, the Appendix reports a catalog containing magnitudes and spectroscopic redshifts for 114 galaxies observed in our first spectroscopic follow-up. The catalog also contains the magnitudes and spectral types for 59 stars in the OACDF.

\section{Field selection and observations}

The central coordinates $(\alpha(2000) \approx 12: 25: 10, \quad \delta(2000) \approx$ $-12: 48: 31)$ of the OACDF were selected in order to identify a field matching the following criteria:

i) lack of sources brighter than $V=9$ mag to minimize CCD saturation and ghost effects;

ii) high galactic latitude to avoid high stellar crowding;

iii) low interstellar (IS) extinction $(E(B-V)<0.03$ mag) according to the Burstein \& Heiles (1982) maps;
Table 1. Coordinates of the OACDF centers.

\begin{tabular}{lllllllll}
\hline \hline Field & \multicolumn{3}{c}{$\alpha(2000)$} & \multicolumn{3}{c}{$\delta(2000)$} \\
\hline OACDF1 & 12 & 26 & 20.4 & -12 & 30 & 20 \\
OACDF2 & 12 & 24 & 27.0 & -12 & 30 & 20 \\
OACDF3 & 12 & 26 & 20.4 & -13 & 01 & 20 \\
OACDF4 & 12 & 24 & 27.4 & -13 & 01 & 20 \\
\hline
\end{tabular}

iv) to be observable from both hemispheres to have the possibility of performing follow-ups using telescopes at several sites.

The observations were carried out in three different periods (18-22 April 1999, 7-12 March 2000 and 26-30 April 2000), using the Wide-Field Imager (WFI) CCD mosaic camera (Baade et al. 1998) at the ESO/MPG $2.2 \mathrm{~m}$ telescope at La Silla in Chile. This camera consists of eight $2 \mathrm{k} \times 4 \mathrm{k}$ CCDs forming a $8 \mathrm{k} \times 8 \mathrm{k}$ array with a scale of $0.238^{\prime \prime} / \mathrm{pix}$.

In the shallow survey, four adjacent $30^{\prime} \times 30^{\prime}$ fields (hereafter OACDF-1 through 4 ) covering a $1^{\circ} \times 1^{\circ}$ field in the sky were observed in the $B, V, R$ and $I$ bands (see Table 1 for the coordinates of the centers of the 4 fields). The OACDF2 and OACDF4 were also selected for the $0.5 \mathrm{deg}^{2}$ deep survey, performed in the $B, V, R$ broad-bands and in the $\lambda 753, \lambda 770, \lambda 791$, $\lambda 815, \lambda 837, \lambda 884$ and $\lambda 914$ medium-band filters.

For the shallow survey, each field was observed in 5 ditherings, while at least 8 ditherings were obtained for the deep survey, the exact number depending on the band. The adopted dithering pattern is that described in the ESO-WFI observers guide (Brewer \& Agustein 2000). A detailed summary of the observations is provided in Table 2.

The standard fields L 98, L 104, L 107, L 110 and PG 1525, selected from the Landolt (1992) equatorial regions, were observed to perform the broad-band photometric calibration. Likewise, the spectrophotometric standard stars EG 274, Hill 600, LTT 3864, LTT 4364, LTT 6248, and LTT 7379 were observed in the medium-band filters for absolute flux calibration purposes. Typically, each standard field was observed twice.

\subsection{Spectroscopic observations}

The first spectroscopic follow-up of the OACDF was performed in April 2000 using the ESO Multi-Mode Instrument (EMMI) at the New Technology Telescope (NTT) in the multiobject spectroscopy (MOS) mode. The selection criteria for the observed objects will be published in our forthcoming paper on the identification and characterization of early-type galaxies in the OACDF. In this paper we report the observations and use the spectra to fine-tune the software parametrization for the photometric redshift determinations and for quality assessment.

Five $9 \times 4$ arc- $\min ^{2}$ fields distributed in the OACDF2 were observed through grism No. 3 , which yields a dispersion of $2.3 \AA$ Aix. Some ten flat-field exposures were taken nightly. Before and after the science spectra, HeAr spectra were observed in each configuration and mask for wavelength calibration purposes. Given the faint magnitude of the targets, the 
Table 2. Summary of the OACDF observations.

\begin{tabular}{|c|c|c|c|c|c|c|c|c|}
\hline Field & Obs. run & Filter & \# diths & $\begin{array}{l}\text { Total exp. } \\
\text { time }\end{array}$ & $\begin{array}{l}\text { Seeing } \\
\text { arcsec }\end{array}$ & Airmass & $\begin{array}{c}\text { Zero points } \\
Z P^{\dagger}\end{array}$ & Col. terms \\
\hline OACDF2 deep & $1 \mathrm{st}$ & $B$ & 12 & $2.0 \mathrm{~h}$ & 1.24 & 1.287 & 24.77 & +0.24 \\
\hline OACDF2 deep & 1 st & $V$ & 10 & $1.7 \mathrm{~h}$ & 1.07 & 1.184 & 24.30 & -0.14 \\
\hline OACDF2 deep & $1 \mathrm{st}$ & $R$ & 13 & $3.3 \mathrm{~h}$ & 1.11 & 1.482 & 24.63 & -0.03 \\
\hline OACDF2 deep & 1 st-2nd & $753 \mathrm{~nm}$ & $9+10$ & $6.5 \mathrm{~h}$ & 0.88 & 1.512 & 21.95 & \\
\hline OACDF2 deep & $1 \mathrm{st}-2 \mathrm{nd}$ & $770 \mathrm{~nm}$ & $9+10$ & $6.0 \mathrm{~h}$ & 0.86 & 1.544 & 21.88 & \\
\hline OACDF2 deep & 1 st-2nd & $790 \mathrm{~nm}$ & $9+10$ & $6.5 \mathrm{~h}$ & 0.99 & 1.084 & 21.80 & \\
\hline OACDF2 deep & 1 st-2nd & $815 \mathrm{~nm}$ & $9+9$ & $6.8 \mathrm{~h}$ & 0.79 & 1.044 & 21.59 & \\
\hline OACDF2 deep & 1 st-2nd & $837 \mathrm{~nm}$ & $9+8$ & $6.6 \mathrm{~h}$ & 0.95 & 1.468 & 21.54 & \\
\hline OACDF2 deep & 1 st-2nd & $914 \mathrm{~nm}$ & $10+10$ & $5.6 \mathrm{~h}$ & 0.90 & 1.046 & 21.15 & \\
\hline OACDF4 deep* & 3rd & $B$ & 8 & $2.0 \mathrm{~h}$ & 0.99 & 1.095 & 24.76 & +0.22 \\
\hline OACDF4 deep & $2 \mathrm{nd}-3 \mathrm{rd}$ & $V$ & 7 & $1.8 \mathrm{~h}$ & 0.95 & 1.042 & 24.23 & -0.18 \\
\hline OACDF4 deep & $2 \mathrm{nd}-3 \mathrm{rd}$ & $R$ & $4+14$ & $4.2 \mathrm{~h}$ & 0.99 & 1.410 & 24.54 & -0.03 \\
\hline OACDF4 deep & $2 \mathrm{nd}-3 \mathrm{rd}$ & $753 \mathrm{~nm}$ & $5+10$ & $4.8 \mathrm{~h}$ & 0.95 & 1.042 & 21.81 & \\
\hline OACDF4 deep & 3rd & $770 \mathrm{~nm}$ & 7 & $2.1 \mathrm{~h}$ & 1.33 & 1.250 & 21.72 & \\
\hline OACDF4 deep & 3rd & $790 \mathrm{~nm}$ & 9 & $3.0 \mathrm{~h}$ & 0.95 & 1.380 & 21.65 & \\
\hline OACDF4 deep & 2nd-3rd & $815 \mathrm{~nm}$ & $5+6$ & $3.5 \mathrm{~h}$ & 0.95 & 1.110 & 21.42 & \\
\hline OACDF4 deep & 3rd & $837 \mathrm{~nm}$ & 7 & $3.3 \mathrm{~h}$ & 1.20 & 1.100 & 21.44 & \\
\hline OACDF4 deep & $3 \mathrm{rd}$ & $914 \mathrm{~nm}$ & 9 & $2.5 \mathrm{~h}$ & 0.81 & 1.110 & 21.05 & \\
\hline OACDF shallow & $1 \mathrm{st}$ & $B$ & 5 & $20 \mathrm{~min}$ & 0.86 & 1.078 & 24.77 & +0.24 \\
\hline OACDF shallow & $1 \mathrm{st}$ & $V$ & 5 & $10 \mathrm{~min}$ & 0.83 & 1.237 & 24.30 & -0.14 \\
\hline OACDF shallow* & 2nd & $R$ & 5 & $10 \mathrm{~min}$ & 1.38 & 1.320 & 24.45 & -0.03 \\
\hline OACDF shallow & $1 \mathrm{st}$ & $I$ & 5 & $10 \mathrm{~min}$ & 0.81 & 1.650 & 23.31 & +0.12 \\
\hline
\end{tabular}

$\dagger$ The reported zero points are those for the first and third observing runs, as the second run was partially non-photometric.

* Due to technical problems it was not possible to calibrate the $B$-band during the third run. Stars on the OACDF4, calibrated during the first observing run, were used as secondary standards. A similar procedure was applied to the shallow $R$-band images of the second run.

pixels in the dispersion direction were re-binned by a factor of two to minimize the noise. The resulting nominal resolution was about $10 \AA(F W H M)$ and the covered spectral range was from about $3800 \AA$ to about $8800 \AA$, but the resulting spectral range for each individual object depends on the position of each individual slit relative to the edges of the mask. To remove cosmic ray hits and increase the $S / N$ ratio, three science exposures of 40 min each were made, amounting to a total of $2 \mathrm{~h}$ exposure per field. Three acquisition images, useful for the selection of the slit positions, were obtained by the NTT team prior to the observations. The other three acquisition images were obtained during the first observing night. Typically 35 slits per mask were used. Three apertures were normally assigned to bright stars for mask alignment purposes. The spectrophotometric standard stars Hil600 and Eg274 were observed with the same instrumental set-up as the objects for flux calibration.

\section{Data reduction}

\subsection{Imaging}

Data reduction was performed using the task mscred in $\mathrm{IRAF}^{1}$. Bias correction, super-flat-fielding and fringing correction were done on a nightly basis.

${ }^{1}$ IRAF is distributed by the National Optical Astronomy Observatories, which is operated by the association of the universities for research in astronomy, Inc., under contract with the National Science Foundation.
For each dithering sequence we chose a reference exposure and computed the astrometric solution for the CCDs in that exposure with reference to an external astrometric catalog. A polynomial fit was then performed between the source positions in the reference CCD and those in the other exposures. As reference astrometric catalogue we use the USNO-A2 catalog (Monet et al. 1998) which allows to achieve an absolute rms accuracy $\sim 0.3^{\prime \prime}$. In order to improve the internal astrometric precision we first used the USNO-A2 catalog as a reference for the $R$-band and the resulting catalog, extracted from the $R$-band stacked image, was then used as reference catalog for the pointings in the other bands. The internal rms accuracy between source positions in different bands is less than $0.1^{\prime \prime}$. In order to correct for transparency variations we also selected a reference image and normalized the others to the same flux level, using sources in the overlap regions. Finally, images were resampled, scaled in flux and co-added using the average sigma clip. More details in the WFI data reduction are provided in Alcalá et al. (2002).

\subsubsection{The photometric calibration}

The broad-band photometric calibration to the JohnsonCousins $B V R_{\mathrm{C}} I_{\mathrm{C}}$ system was performed using the standard Landolt (1992) fields. For the field Landolt-98, we used the larger set of photometric standards by Stetson (2000). Photometric calibrated magnitudes and instrumental magnitudes are related by the following equations:

$B=b+C_{B} \cdot(B-V)+Z_{B}$, 
$V=v+C_{V} \cdot(B-V)+Z_{V}$,

$R_{\mathrm{C}}=r+C_{R} \cdot\left(V-R_{\mathrm{C}}\right)+Z_{R}$,

$I_{\mathrm{C}}=i+C_{I} \cdot\left(V-I_{\mathrm{C}}\right)+Z_{I}$,

where $b, v, r, i$ are the instrumental magnitudes corrected for atmospheric extinction, $C_{B}, C_{V}, C_{R}, C_{I}$ the color terms and $Z_{B}, Z_{V}, Z_{R}, Z_{I}$ the zero points. We used the mean extinction coefficients for the La Silla observatory ${ }^{2}$.

Photometric calibrations were achieved using a number of stars ranging from 100 to 200 . The fit residuals are typically less than $3 \%$, but in some cases the residuals are as high as $5 \%$. The relatively high residuals may be a consequence of the nonuniform background illumination over the field of view, that will be discussed in Sect. 3.2. A few points with particularly high residuals are due to stars partially falling in the CCD gaps and/or to photometric/astrometric errors in the standard cata$\log$. Aberrant points were rejected by a sigma clipping algorithm. Points typically above the $2 \sigma$ level were not used for the calibration.

The photometric zero points and the corresponding color terms are listed in Table 2. Since the second run was partially non-photometric, we give in Table 2 only the zero points of the first and third observing runs. Also, due to technical problems, it was not possible to calibrate the $B$-band of the field OACDF4 during the third observing run. The latter calibration was done using secondary standard stars in the OACDF. A similar approach was adopted for the shallow observations of the second observing run in the $R$-band.

The flux calibration for the medium-band observations was performed using spectrophotometric standard stars. These calibrations provide $A B$ magnitudes. The detailed explanation of the medium-band filter calibrations, as well as the determination of $A B$ magnitudes can be found in Alcalá et al. (2002).

For practical purposes, we transform our broad-band instrumental WFI magnitudes into $A B$ magnitudes using the following expressions:

$B_{A B}=b+Z_{B}-0.093$,

$V_{A B}=v+Z_{V}+0.002$,

$R_{A B}=r+Z_{R}+0.215$,

$I_{A B}=i+Z_{I}+0.509$.

In the catalog presented in the appendix, $A B$ magnitudes are given (Tables A.1 and A.2).

In order to check the photometric homogeneity over the eight CCDs and its dependence on the star color (Manfroid \& Selman 2001; Monelli et al. 2003), we also observed a few selected standard fields in each of the eight CCDs. For the broadband filters, we used the Landolt field PG1525-071, which contains five stars with $(B-V)$ colors ranging from -0.198 to 1.109 . For the medium-band filters we used the spectro-photometric

${ }^{2}$ See http://obswww. unige.ch/photom/extlast.html and http://www.1s.eso.org/lasilla/sciops/2p2/D1p5M/misc/ Extinction.html standards Eg 274, Hill 600 and LTT 6248. We find no color dependence as well as no time dependence but, on the other hand, we find an offset of the instrumental magnitude among the eight CCDs. The effect is summarized in Fig. 1, where we show the fluxes of the standard stars measured in each $\mathrm{CCD}$, relative to the mean value over the $8 \mathrm{CCDs}$. Within the errors, the trend is the same in the different bands $(B, V, R, I)$ and at different times (from April 1999 to April 2000). As stressed by the above quoted authors this photometric offset may be attributed to an additional light pattern caused by internal reflections of the telescope corrector. Such a pattern, with an amplitude that depends also on the exposure time, is present in each image (including flat-fields) and must be corrected before flat-fielding. In order to correct for this effect in a proper way, one should know the additional-light pattern.

The photometric offset appears to be slightly stronger in the medium-band filters than in the broad-band ones (see Fig. 1 right panel). We find an offset in the flux of the stars which may introduce an average uncertainty of $\pm 3 \%$ in the broad-band $B$, $V, R, I$ filters and of $\pm 5 \%$ in the medium-band filters.

\subsection{Spectroscopy}

The MOS data reduction was performed using the MIDAS package following standard reduction techniques for multiobject slit spectroscopy. For each configuration or mask, the reduction consisted of the bias subtraction, definition of slit positions, flat-fielding, row-by-row wavelength calibration, background subtraction, extraction of one-dimensional spectra and relative flux calibration.

For each mask or field, the reduction steps were the following:

i) the average bias frame was subtracted from all the frames and a master flat-field was determined using the median option within MIDAS;

ii) the definition of the slit positions (along the direction perpendicular to the dispersion) and the flat-fielding were performed using the master flat and the context MOS within the MIDAS environment;

iii) the three multi-spectra frames of each field were combined using a sigma clipping algorithm for the rejection of cosmic ray hits;

iv) for each configuration or mask, the individual twodimensional long-slit spectra were extracted from the combined multi-spectra frames. The next steps were then performed following the standard pipeline of the context "long" within the MIDAS package;

v) a row-by-row wavelength calibration was done on each individual two-dimensional long-slit spectrum using its corresponding HeAr comparison spectrum, extracted exactly in the same way as the science spectrum;

vi) the background subtraction was then performed and the one-dimensional spectra were extracted;

vii) the spectra of the flux standards, reduced in the same way as the science spectra, were then used for the determination of a mean response function. A response function was determined for each one of the two observing nights. 

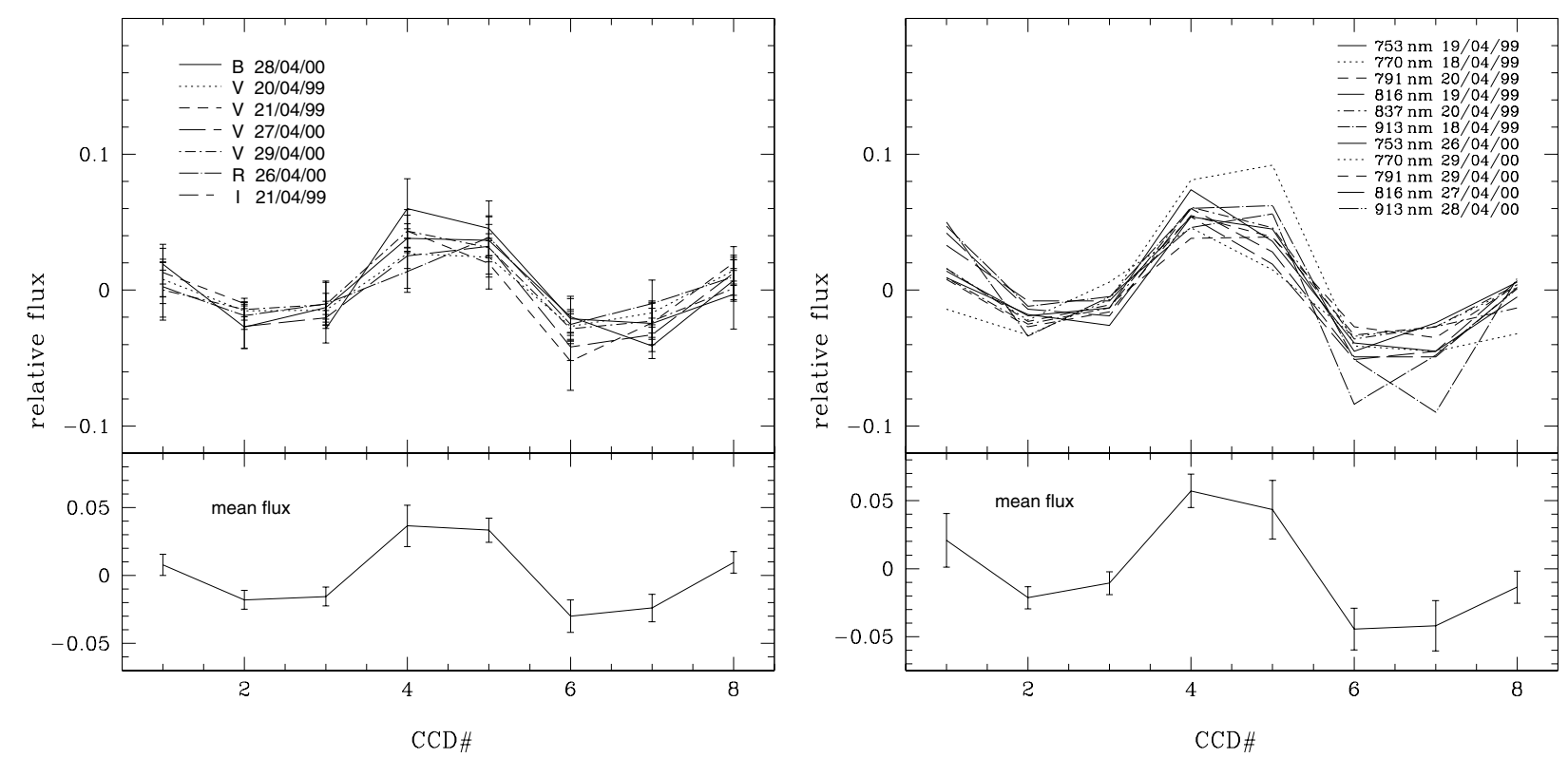

Fig. 1. Left: relative photometric offsets versus the CCDs for the broad-band filters (upper panel). The different line types represent the different observing dates as labeled. The average over the broad-band filters is shown in the lower panel. Right: the same but for the medium-band filters.

Finally, a relative flux calibration was then performed on the one-dimensional spectra by applying the corresponding response function.

\section{Source extraction from the WFI images}

Catalog extraction was performed using SExtractor (Bertin \& Arnouts 1996). In order to "increase" the signal-to-noise ratio, the images are filtered with a kernel that, in our case, is a Gaussian with a constant $F W H M$ that properly matches the seeing on the images. The source detection is performed on the filtered image and the detection threshold is determined as the best compromise between limiting magnitude and percentage of spurious detections.

The photometry was also performed with SExtractor. Magnitudes were measured both in fixed circular or CORE apertures ( $2 \sigma$ and $3 \sigma$ of the PSF FWHM) and in Kron-like (1980) elliptical apertures (MAG_AUTO in SExtractor, cf. Bertin \& Arnouts 1996).

MAG_AUTO provides the best measurement of the object total flux because the elliptical aperture is adaptively determined from the object dimensions. However, in the case of blended objects MAG_AUTO does not provide reliable results. In these cases (indeed representing a very low percentage of the total catalogue), we use the CORE aperture photometry.

We also use the MAG_AUTO magnitudes to measure objects colors, except in the case of blended sources in which CORE aperture magnitudes are used.

\subsection{Completeness magnitude limit of the OACDF}

The best parameter set for the catalog extraction were determined, for each final stacked image, using a series of Monte Carlo simulations. The goal was: $i$ ) to determine the completeness limit of the extracted catalogs; and ii) to estimate of the fraction of spurious detections (see Arnaboldi et al. 2002, for details).

We ran a series of simulations by randomly adding a synthetic population of point-like sources with a known luminosity fuction (LF) and positions. The completeness limit, i.e. the magnitude at which $50 \%$ of the input catalog is lost, was then recovered by matching the extracted catalog with the modelled population. This procedure allowed us to derive the completeness limit of 25.1 AB mag for the $R$-band mosaic (see Fig. 2). The completeness limits for the OACDF at $S / N$ ratios of 10 and 5 in the different bands are listed in Table 3.

In order to estimate the fraction of spurious detections we proceeded as follows: a simulated image was built from the background interpolated by SExtractor, by adding the noise map constructed by multiplying the SExtractor rms map by a Gaussian noise image with null mean and $\mathrm{rms}=1$. For a given SExtractor parameter set, the number of spurious detections was then determined as the difference between the SExtractor catalog and the matched catalog with the input modelled population. For the OACDF $R$-band mosaic, we find that the number of spurious sources, relative to the total number of detected objects, is less than $2 \%$ at the completeness limit of $25.1 \mathrm{AB}$ mag.

Besides the usual problems posed by the bright (saturated) stars which produce a large number of spurious objects in their extended halos, mosaic images pose additional problems in the regions at the edges of the individual images which have lower $S / N$ due to the dithering. The usual approach would have been to mask out these regions, keeping track of their size and position to correct the final statistics. However, so as not to lose completely the information on the objects falling in such regions, a flag was introduced in the catalogues as follows: we flag as "BAD" those objects having either high background values (i.e. objects in proximity of saturated objects) or high background rms values (i.e. mostly border objects). The threshold to activate such flag was optimized on a trial and error 
Table 3. Completeness magnitudes vs. wavelengths.

\begin{tabular}{cccccccccc}
\hline \hline$S / N$ & $B_{A B}$ & $V_{A B}$ & $R_{A B}$ & 753 & 770 & 791 & 816 & 837 & 915 \\
& & & & & & & & & \\
\hline 10 & 24.6 & 24.0 & 24.3 & 22.8 & 22.4 & 22.1 & 22.5 & 21.8 & 21.9 \\
5 & 25.3 & 24.8 & 25.1 & 23.7 & 23.3 & 23.0 & 23.4 & 22.7 & 22.8 \\
\hline
\end{tabular}

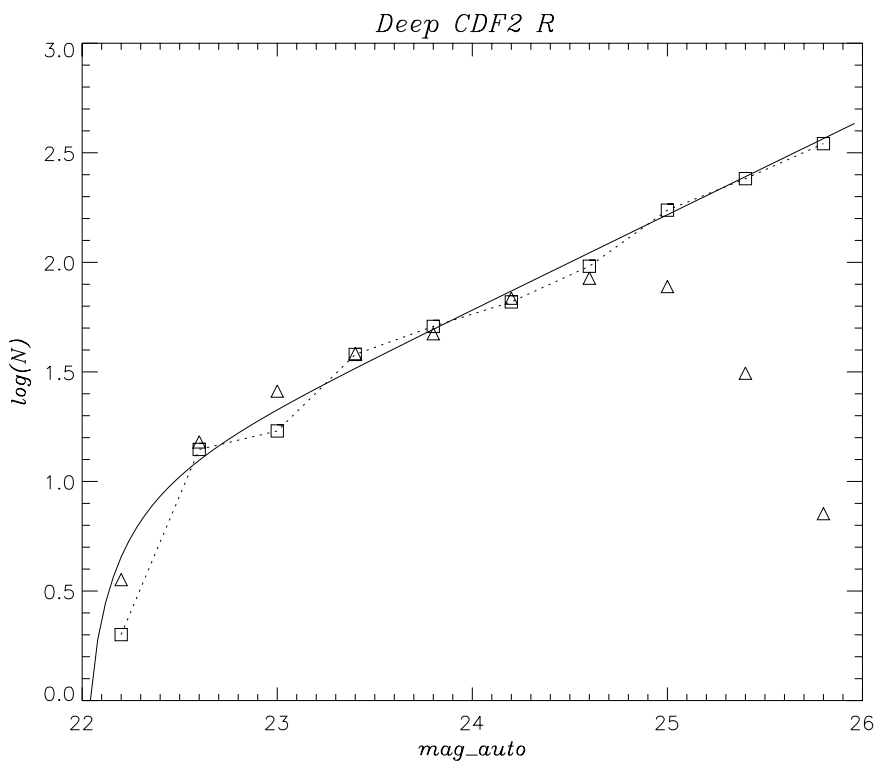

Fig. 2. Magnitude distributions in the $R$-band mosaic. The simulated sample, connected by the dotted line, is represented with squares, while the extracted sample is represented with triangles. The solid line represents the magnitude distribution of the modeled sample.

basis via the accurate inspection of objects flagged as spurious. Extensive testing showed that the best compromise was achieved by setting such treshold at either 5 times the value of the local background images and/or at a local rms $20 \%$ larger than the average rms of the mosaic itself. These criteria assume that the catalogs have been extracted from background subtracted images.

\subsection{Star-galaxy separation}

To distinguish point-like sources from extended ones we use the SExtractor parameter CLASS_STAR. Star/galaxy classification in SExtractor is made using neural networks trained on simulated galaxies on single CCD images and is mainly based on the deviations of extended objects from a well behaved PSF. We use different CLASS_STAR indexes for the different bands. We set CLASS_STAR equal to $0.95,0.98$ and 0.98 for the $B, V$ and $R_{\mathrm{C}}$ bands respectively.

In mosaic images, the determination of the final PSF is usually non trivial due to the combined effects of ditherings and varying PSF among the single images. In order to test the reliability of the SExtractor CLASS_STAR parameter we use the spectra obtained for the sample of 173 objects (114 galaxies and 59 stars). In this sampe, the objects classified as stars by SExtractor are 63 with $5 \%$ contamination due to misclassified galaxies, while the objects classified as galaxies are 110 with no contaminants. Overall, the SExtractor classification turns out to be quite reliable in the magnitude range of the spectroscopic sample, i.e. brighter than $I=22$.

\section{Quality assessment}

In the following sub-sections we assess the quality of the OACDF catalogs by the comparison of the galaxy number counts with those in the literature and using the spectroscopic observations of stars and galaxies in the OACDF.

\subsection{Source counts}

In Fig. 3 the number counts of galaxies in the OACDF, in the $B, V, R_{\mathrm{C}}$ deep fields, are shown. Other number counts from the recent literature are also overplotted for comparison. The star/galaxy separation is done here on each single catalog by using the stellarity index from SExtractor, but without completeness correction applied. Despite this, there is a good agreement between the source number counts of the OACDF data and those from the literature.

The number counts $\left(\log N / 0.5 \mathrm{mag} / \mathrm{deg}^{-2}\right)$ per magnitude bin at each band are reported in Table 4 . The magnitudes are $A B$ magnitudes. The logarithmic counts are reported in halfmagnitude bins, selected in the $B, V$, and $R$ bands, normalized to the effective area of the OACDF deep survey $\left(0.5 \mathrm{deg}^{2}\right)$.

\subsection{Spectroscopic redshifts}

In order to determine spectroscopic redshifts the $\mathrm{Ca}$ II at $\lambda 3933.68$ and $\lambda 3968.49 \AA$ lines and the $\operatorname{Mgb}(\lambda 5173 \AA)$ absorption features, as well as the " $G$ " band at $\lambda 4300 \AA$ were identified and fitted with Gaussians for the central wavelength measurements. In order to further confirm the redshift determinations, a cross-correlation analysis was applied to the sample of galaxy spectra. For this purpose we used the fxcor task under IRAF. Several late type stars, obtained with the same instrumental set up, were used as templates for the cross-correlation analysis. Moreover, only parts of the spectra, not affected by telluric absorption lines, were considered. The typical error of the spectroscopic redshifts is around \pm 0.005 .

The total number of useful spectra is 173 . The coordinates, $A B$ magnitudes, redshifts and spectral types (see next section) of these objects are listed in Tables A.1-A.3. 59 of these spectra resulted to be stellar objects. The remaining 114 objects are galaxies. For 24 of these, the redshift determination is uncertain because their $S / N$ ratio is less than 10 . We distinguish these objects in Table A.3 with a question mark next to the redshift value. 

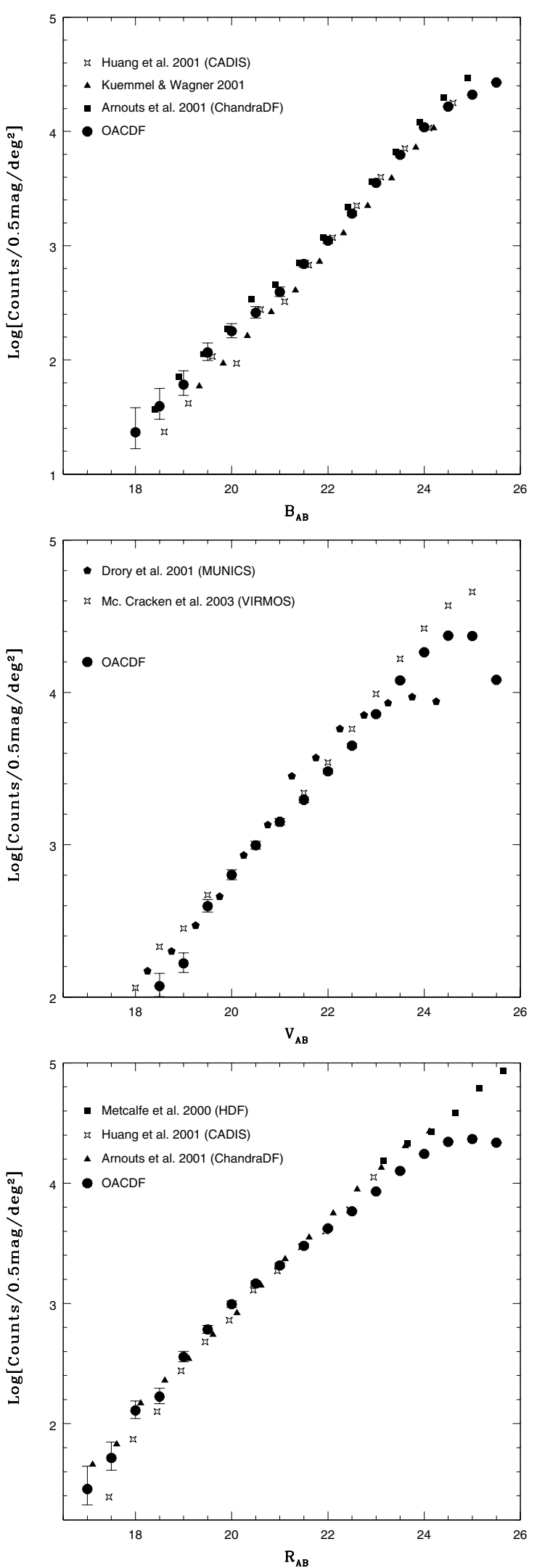

Fig. 3. Comparison of the source number counts in the OACDF (filled circles) with those from the literature. The logarithmic counts are in half-magnitude bins, in the $B, V$, and $R$ bands (top, middle and bottom panels respectively) and are normalized to the OACDF deep survey area of $0.5 \mathrm{deg}^{2}$. The $1 \sigma$ Poissonian errors, also provided in Table 4 , are shown.
Table 4. Source counts of the OACDF at each band.

\begin{tabular}{cccc}
\hline \hline AB mag. & $\log N_{B} \mathrm{deg}^{-2}$ & $\log N_{V} \mathrm{deg}^{-2}$ & $\log N_{R}$ deg \\
\hline 17.0 & $0.90_{-0.53}^{+0.23}$ & $1.10_{-0.33}^{+0.19}$ & $1.46_{-0.19}^{+0.13}$ \\
17.5 & $1.00_{-0.37}^{+0.20}$ & $1.38_{-0.19}^{+0.13}$ & $1.72_{-0.13}^{+0.10}$ \\
18.0 & $1.35_{-0.22}^{+0.14}$ & $1.74_{-0.13}^{+0.10}$ & $2.11_{-0.08}^{+0.07}$ \\
18.5 & $1.60_{-0.16}^{+0.11}$ & $2.10_{-0.08}^{+0.07}$ & $2.23_{-0.07}^{+0.06}$ \\
19.0 & $1.77_{-0.12}^{+0.09}$ & $2.26_{-0.07}^{+0.06}$ & $2.56_{-0.05}^{+0.04}$ \\
19.5 & $2.05_{-0.08}^{+0.07}$ & $2.62_{-0.04}^{+0.04}$ & $2.78_{-0.03}^{+0.03}$ \\
20.0 & $2.26_{-0.07}^{+0.06}$ & $2.81_{-0.03}^{+0.03}$ & $2.99_{-0.03}^{+0.03}$ \\
20.5 & $2.42_{-0.06}^{+0.06}$ & $3.00_{-0.03}^{+0.02}$ & $3.17_{-0.02}^{+0.02}$ \\
21.0 & $2.59_{-0.04}^{+0.04}$ & $3.17_{-0.02}^{+0.02}$ & $3.32_{-0.02}^{+0.02}$ \\
21.5 & $2.86_{-0.03}^{+0.03}$ & $3.30_{-0.02}^{+0.02}$ & $3.48_{-0.01}^{+0.02}$ \\
22.0 & $3.05_{-0.02}^{+0.02}$ & $3.49_{-0.02}^{+0.01}$ & $3.62_{-0.01}^{+0.01}$ \\
22.5 & $3.29_{-0.02}^{+0.02}$ & $3.65_{-0.01}^{+0.01}$ & $3.77_{-0.01}^{+0.01}$ \\
23.0 & $3.54_{-0.01}^{+0.01}$ & $3.86_{-0.01}^{+0.01}$ & $3.93_{-0.01}^{+0.01}$ \\
23.5 & $3.79_{-0.01}^{+0.01}$ & $4.08_{-0.01}^{+0.01}$ & $4.10_{-0.01}^{+0.01}$ \\
24.0 & $4.03_{-0.01}^{+0.01}$ & $4.26_{-0.01}^{+0.01}$ & $4.24_{-0.01}^{+0.01}$ \\
24.5 & $4.21_{-0.01}^{+0.01}$ & $4.37_{-0.01}^{+0.01}$ & $4.34_{-0.01}^{+0.01}$ \\
25.0 & $4.31_{-0.01}^{+0.01}$ & $4.35_{-0.01}^{+0.01}$ & $4.37_{-0.01}^{+0.01}$ \\
25.5 & $4.41_{-0.01}^{+0.01}$ & $4.06_{-0.01}^{+0.01}$ & $4.34_{-0.01}^{+0.01}$ \\
\hline & & & \\
& & &
\end{tabular}

\subsection{Stellar spectral types}

The stellar spectral types were determined by visual comparison of the stellar spectra with those of the grid of spectral standard stars taken from the library of stellar spectra by Jacoby et al. (1984). The most important spectral features considered for the spectral type classification were: $\mathrm{Mg}$ I $\lambda 5167, \lambda 5173$, and, whenever possible, the " $G$ " band $\lambda 4300$ for the G stars, the $\mathrm{Ca} I \lambda 6162$ and $\mathrm{Fe} \mathrm{I} \lambda 5227$ for the late G and early K stars, the Na I $\lambda 5893$ and the Fe I blend $\lambda 6495$ for the K stars, and the $\mathrm{TiO}$ bands $\lambda 4954, \lambda 5448, \lambda 6159$ for the late $\mathrm{K}$ and the $\mathrm{M}$ stars.

The magnitudes and spectral types of these stars are provided in Tables A.1-A.3. In order to transform the $B_{A B}$, $V_{A B}, R_{A B}$ and $I_{A B}$ magnitudes, reported in Table A.1, into the Johnson-Cousins system, the transformation Eqs. (9)-(12) given in the appendix, must be applied.

In Fig. 4 the $(B-V)$ and $\left(V-I_{\mathrm{C}}\right)$ colors of the stars are plotted versus their spectral types. In these plots the relation between color versus spectral type, taken from the stellar library by Lejeune et al. (1997), is over-plotted as a continuous line. As can be seen, there is a good agreement of the spectral types and colors with those of the library of stellar spectra by Lejeune et al. (1997).

As a further check, in Figure 5 we show the $(B-V)$ vs. $\left(V-R_{\mathrm{C}}\right)$ diagram of point-like sources (CLASS_STAR $\left.>0.85\right)$ in the OACDF. As expected, the colors are consistent with those expected for stars, which provides a further photometric check of the OACDF catalogs.

\subsection{Photometric redshifts}

Photometric redshifts were determined using the HYPERZ package (see Bolzonella et al. 2000 for details). 

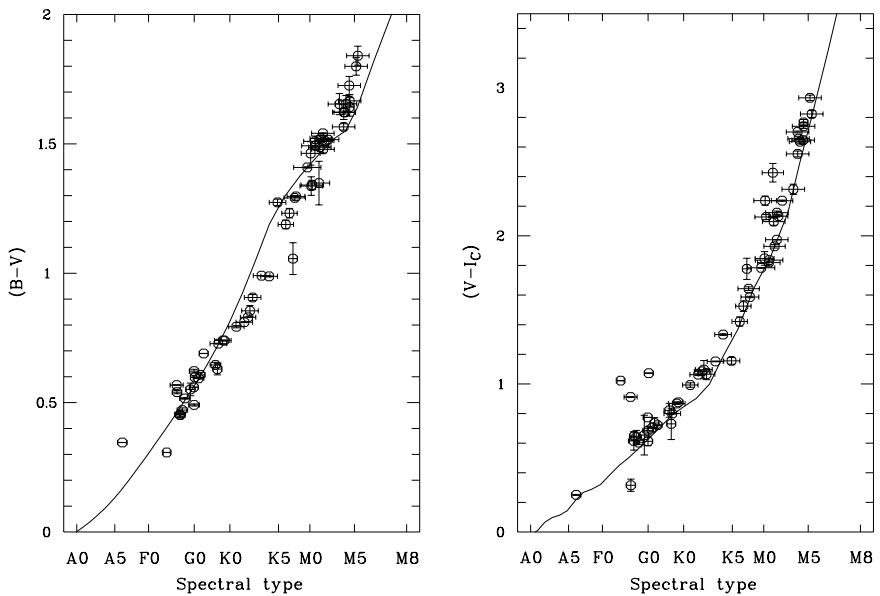

Fig. 4. $(B-V)$ and $\left(V-I_{\mathrm{C}}\right)$ colors of the stars observed with EMMI-MOS versus their spectral type (circles in the left and right panels respectively). The continuous line represents the relationship between the corresponding colors and spectral type, taken from the stellar library by Lejeune et al. (1997).

The spectroscopic redshifts were used to fine-tune the software parametrization. The optimal set-up, which yields the most consistent results with the spectroscopic redshifts, was to use three broad-bands $(B, V, R)$ plus the medium-band filters centered at 753 and $914 \mathrm{~nm}$. We use the MAG_AUTO magnitudes (see Sect. 4) for the photometric redshifts computation because they provide more robust results for all type of objects in the catalog.

The procedure we used for the photometric redshift determinations does not include templates for QSOs. Therefore, the results for objects like OACDF122436.7-124519, which is a relatively high-redshift QSO $(z \sim 3)$, are also unreliable. For this particular case, HYPERZ provides a photometric redshift of $z_{\text {phot }}=0.23$. In forthcoming papers, we will include AGN-like templates in order to minimize such large residuals.

The spectroscopic redshifts versus the photometric redshifts of different types of objects in the OACDF2 are plotted in Fig. 6. The black dots represent the galaxies with well determined spectroscopic redshifts, while the open circles represent the 24 galaxies with dubious redshift determinations (indicated with a question mark next to the spectroscopic redshift in Table A.3).

Despite the dubious redshifts, the vast majority of the photometric redshifts match well the spectroscopic redshifts within the errors. Six out of 113 objects fall off the general trend (a percentage compatible with that of previous works). We find that the mean difference between photometric and spectroscopic redshifts is $0.02 \pm 0.07$ (see Fig. 6 right panel). Therefore, our photometric redshift determinations are confident within an rms error of about 0.07 , in the redshift range from 0 to 0.7 .

The distribution of photometric redshifts of the OACDF, is shown in Fig. 7. The distribution of the CFRS (Canada France Redshift Survey, Lilly et al. 1995) is over-plotted with a dashed line for comparison.

For the sake of this comparison, we have selected two classes of objects from the whole catalog: $i$ ) the bright class

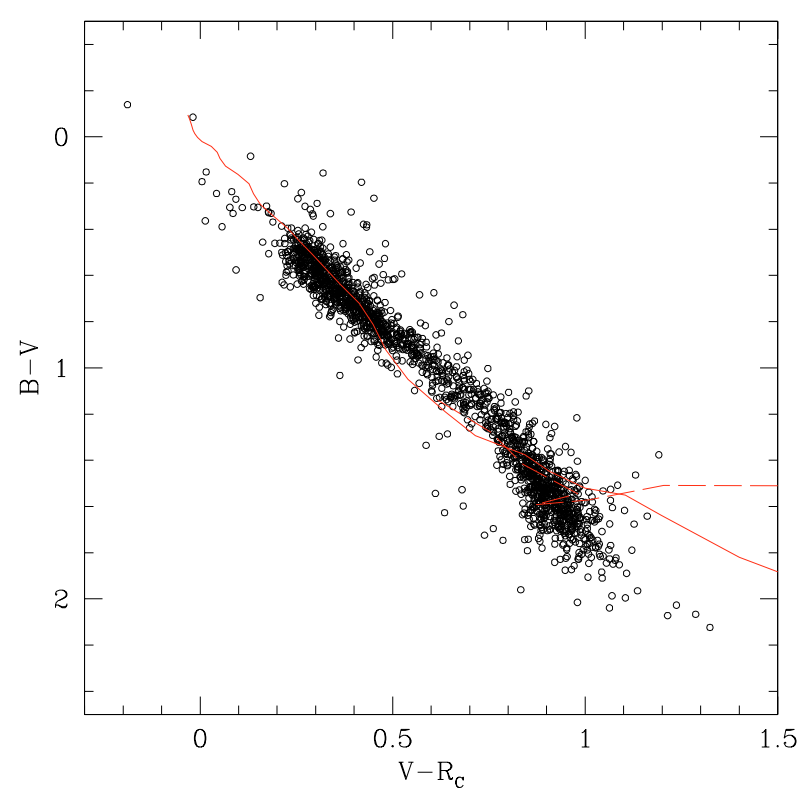

Fig. 5. Color-color diagram for the objects classified as stars (open circles) using the criterium CLASS_STAR $>0.85$ for the sources detected in the $B, V$ and $R$ bands. The synthetic colors for dwarfs and giants, taken from the standard stellar library by Lejeune et al. (1997), are plotted with the continuous and dashed lines respectively.

is composed by objects with a high photometric $S / N$ ratio and hence, objects for which the results obtained from the comparison with the spectroscopic follow-up are consistent; $i i)$ the faint class, i.e. all the objects fainter than $V_{A B}=22 \mathrm{mag}$. Although the latter were detected with a $S / N>10$, we still flagged them because we do not have any spectroscopic confirmation of their redshifts. The figure shows the objects in the bright class only.

In order to confirm the similarity of the two redshift distributions shown in Fig. 7 we performed a Kolmogorov-Smirnov two sample test (KS test), which is a non-parametric test that yields correct probabilities without conditions for the distribution of the errors. The result of the KS test is that the two redshift distributions are similar at the $99.4 \%$ confidence level. We can thus conclude that the two distributions come from the same parent distribution.

\section{Search for groups and clusters of galaxies}

An accurate knowledge of the global properties of galaxy clusters and groups is needed both to constrain the models of galaxy formation and evolution and to falsify the cosmological models. The first step in the study of galaxy clusters is, however, the construction of statistically significant and unbiased catalogues of cosmic structures spanning a wide range of richness $(N)$ : from very low multiplicity structures such as galaxy triplets $(N=3)$, to groups $(N<50)$, and clusters $(N>50)$. While much work has been devoted to the construction of complete rich cluster samples, the low multiplicity end of the structure spectrum is still poorly known due to the difficulties encountered in identifying physically bound systems of low multiplicity on wide field or survey material. On projected data, in fact, loose and poor groups of galaxies produce low signal/noise 

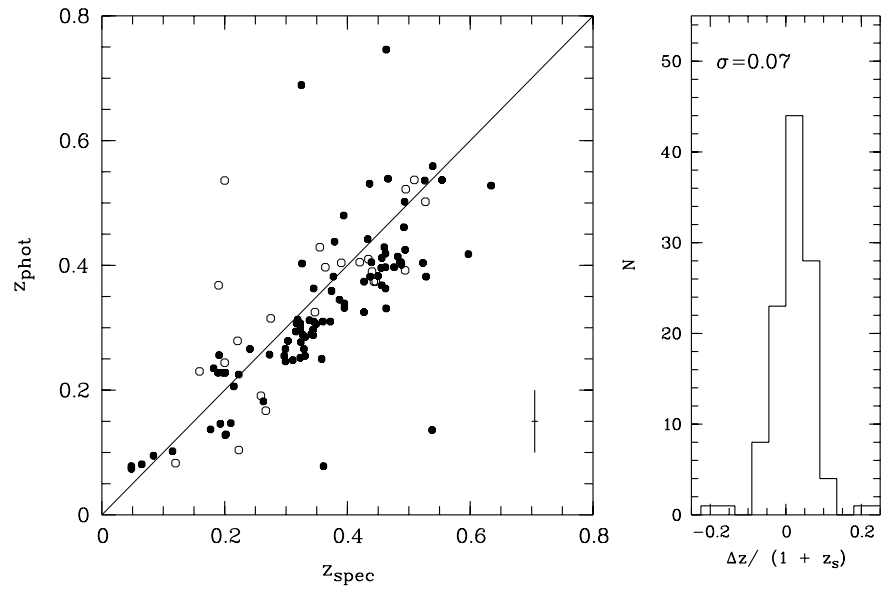

Fig. 6. Left panel: photometric versus spectroscopic redshifts for the objects reported in Table A.3. The black dots represent the galaxies with well determined spectroscopic redshifts, while the open circles represent the 24 galaxies with dubious redshift determinations (indicated with a "?" next to $z_{\text {spec }}$ in Table A.3). The continuous line represents the relation $z_{\text {phot }}=z_{\text {spec }}$; right panel: distribution of the difference $z_{\text {spec }}-z_{\text {phot }}$.

overdensities which are difficult to detect on shallow photometric material and require spectroscopic surveys and/or deep fields covering moderately wide areas.

Not so much work has been done to detect poor structures such as loose groups; two principal methods (and their successive elaborations) have been adopted. In particular, Turner \& Gott (1976) presented the first tentative objective identification of groups as enhancements above a reliable threshold in the projected galaxy distribution. A noticeable exception to the lack of low-richness catalogs has been the detection of compact groups, where several teams (de Carvalho \& Djorgovski 1995; Iovino et al. 1999, 2003) have proposed different approaches to their detection. For the determination of the mass function, it is important to note that its derivation from the above-cited catalogues is hindered by the fact that all of the above algorithms are optimized for the detection of either groups or clusters, and with a few exceptions (cf. Puddu et al. 2003) no systematic work has been done in matching their outcomes in the transition region between structures of low and high richness. The OACDF, as any other deep field, offers the possibility to begin covering this gap. In fact, by assuming an Euclidean metric and uniform distribution we expect to find in the OACDF ca. 5 groups in the richness range [5, 30] (against less than one rich cluster). Since the investigated area of the OACDF is limited to $0.5 \mathrm{deg}^{2}$, we expect to find about 4 groups in the richness range $[5,30]$ and less than one cluster in the richness range $[30,100]$, in the volume enclosed within $0.5 \mathrm{deg}^{2}$ and $z<1.0$.

In order to actually detect them we used the method developed by Puddu et al. (2003) and already applied to the DPOSS data (about $300 \mathrm{deg}^{2}$; see Puddu et al. 2001). In summary: we first extracted a catalogue containing all extended sources with $20.5<R<24$ and then binned the corresponding galaxy spatial distribution into equal area square bins to produce a density map, such that the mean number of galaxies per bin is $\sim 1$ (binsize $=0.005 \mathrm{deg}$ ). In order to identify and extract the density

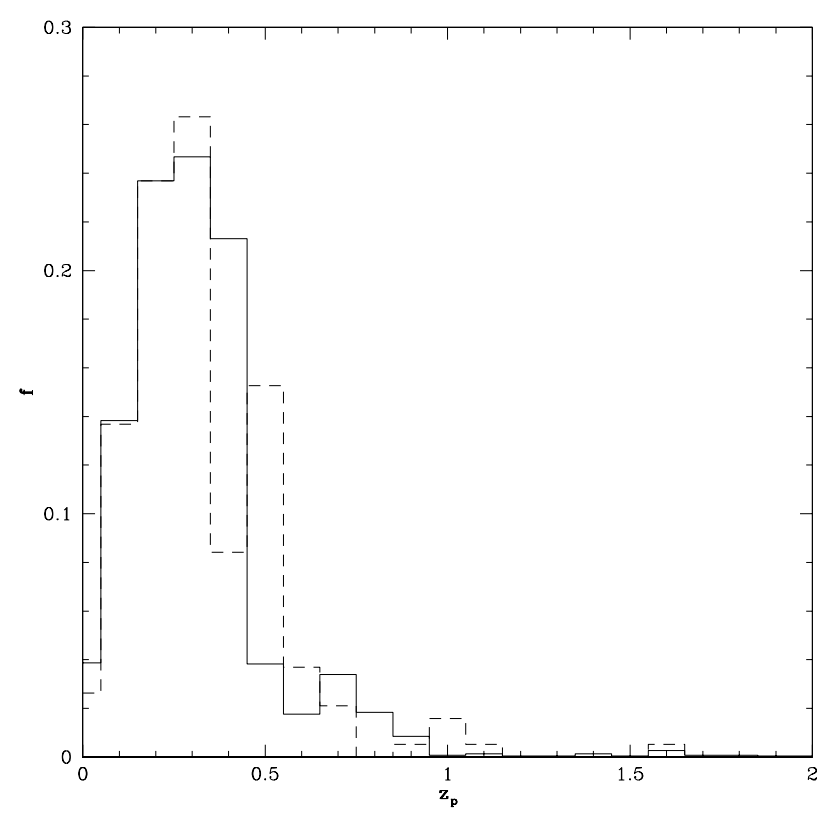

Fig. 7. Distribution of photometric redshifts of the high $S / N$ OACDF catalog (continuous line) compared with the redshift distribution of the CFRS (dashed line) at the same depth.

peaks on the resulting density map, we run SExtractor (after background independent estimation and subtraction) with an absolute threshold of 0.5 (objects/pixel) and a minimum detection area of 4 pixels. The background subtraction removes all the structures with spatial frequencies smaller than the scale length of 50 pixels, which corresponds to linear dimensions of 3-4 Mpc, in the redshift range 0.3,0.6. For the background galaxies, we find a mean density of $\sim 43000 \pm 4200$ objects per square degree. In order to enhance the structures, the detection was ran on the density map convolved with a Gaussian filter 3 pixels wide (corresponding to $150-250 \mathrm{kpc}$, at redshift 0.3-1.0). The latter are reliable dimensions for a cluster core. A caveat of our method is that the Gaussian filter sets strong limitations on the possibility of detecting structures at higher redshifts, which appear with a more compact core than the minimum dimension of the filter itself. The over-density regions are defined by the isodensity contours corresponding to the detection threshold on a smoothed map (Fig. 8). The area defined by these contours is called the iso-area. We used the number of objects lying inside this area to give an estimate of the cluster candidate richness. The error on the richness estimate depends on the $S / N$ ratio of the detection and is inversely correlated with the richness itself: low and intermediate richness $(<30)$ candidates have a low detection $S / N$ and hence are not detected with an high confidence. We then use colour-magnitude diagrams to look for a possible red sequence of early type galaxies, confirming that the overdensity corresponds to a galaxy cluster.

The cluster red sequence can be isolated from the background objects using a pair of filters, if these filters sample the $4000 \AA$ break. In our case we chose the $\left(V-R_{\mathrm{C}}\right)$ color and, in order to explore a larger redshift range, we also use the $V-913$ color. There is, however, the caveat that the limiting magnitude in the narrow band filters is brighter than for the $V$ and $R_{\mathrm{C}}$ bands with the consequence that not all the 


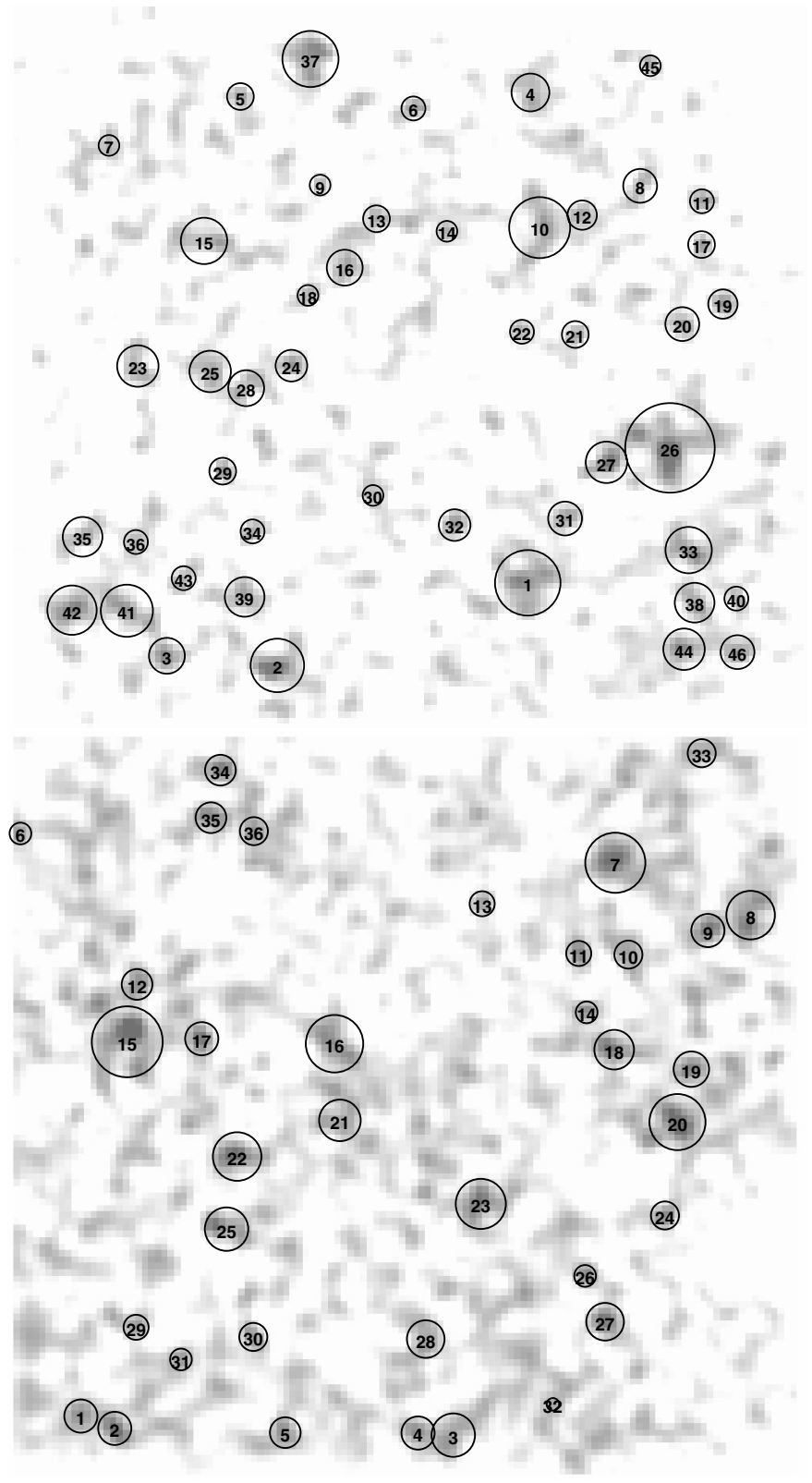

Fig. 8. Smoothed density maps of the OACDF2 (top) and OACDF4 (bottom) fields. Each map has been produced by binning the matched catalogs (in the $B, V$ and $R$ bands) into equal area square bins of $18 \times 18$ squared arc-sec (see Puddu et al. 2003 for details). The circles mark the detected over-densities (including those having a low $S / N$ ratio).

objects present in the $\left(V-R_{\mathrm{C}}\right)$ sample will be retrieved in the $V-913$ sample. However, this is not a problem for our analysis because we are interested in the bright ellipticals of the red sequence rather than in faint background galaxies.

For each over-density, we plot the color-magnitude diagrams $\left(V-R_{\mathrm{C}}\right)$ vs. $R_{\mathrm{C}}$ and $V-913$ vs. 913 of all the objects in an aperture with an area equivalent to three times the iso-area and centered on the over-density centroid. We then select a comparison field in an annular region centered on the cluster, with a much larger radius, but with comparable area as that defined by the over-density. We then perform a statistical subtraction of

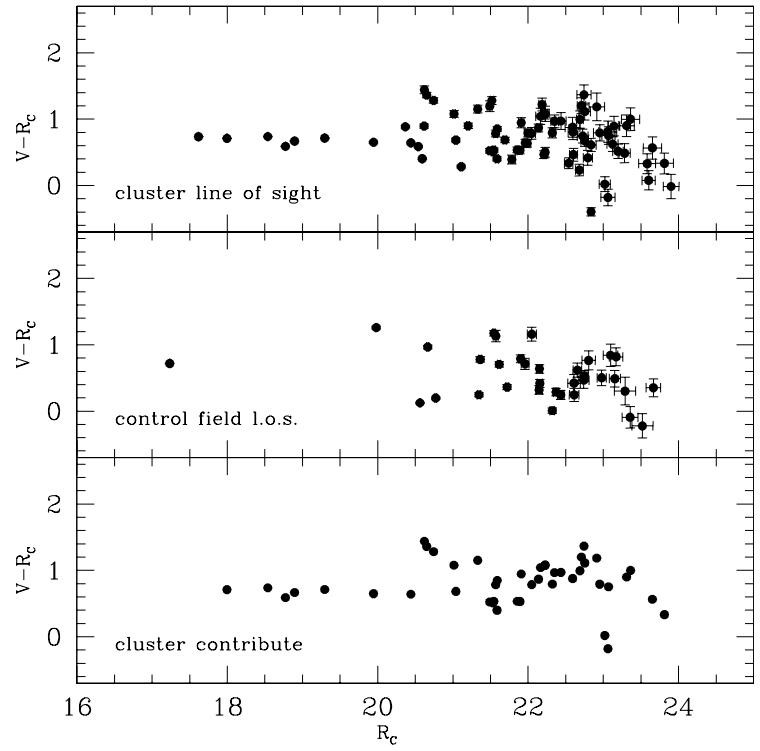

Fig. 9. Example of the statistical subtraction applied to the cluster candidate OACDF2_2. The $\left(V-R_{\mathrm{C}}\right)$ vs. $R_{\mathrm{C}}$ diagram for all the objects (cluster plus background) in the over-density region is shown in the upper panel, while the same diagram for the background objects is shown in the middle panel; the results after the statistical subtraction are shown in the bottom panel.

the background contribution, which consists on the elimination of the points nearest to the neighbor background points in the color-magnitude diagram. In this way, the red sequence is enhanced, if it is really present. As an example, we show in Fig. 9 the procedure applied to the cluster candidate OACDF-CLG02.

Ten candidates for clusters of galaxies were selected. However, we note that a large number of low $S / N$ overdensities, that coincide with many groups of galaxies, is detected in the OACDF. In Fig. 10 we plot the $\left(V-R_{\mathrm{C}}\right)$ vs. $R_{\mathrm{C}}$ and $V-913$ vs. 913 color-magnitude diagrams of our four best cases selected on the OACDF2 and OACDF4 after the statistical subtraction of the background objects on the colormagnitude plane.

The coordinates, estimated richness, iso-area (in pixels), $S / N$ ratio and the estimated photometric redshift for a list of galaxy cluster candidates, detected with $S / N>3$ and selected on the basis of their red sequences, is provided in Table 5. The coordinates correspond to the over-density centroids. The richness estimate was done as described above. The photometric redshift for each candidate was estimated as the median of the photometric redshifts of the objects in the corresponding aperture (three times the iso-area).

It is interesting to note that the color-magnitude diagram of the cluster candidate OACDF-CLG02 shows some hint of a double red sequence, which is more evident in the $V-913$ vs. 913 diagram. This leads to the idea of two galaxy clusters at different redshifts (one at $z=0.23$ and the other one at $z \approx 0.6$ ) which by chance projection fall approximately along the same line of sight. The spectroscopic observations reported in Sect. 5 confirm the first galaxy cluster at $z=0.20$. This 

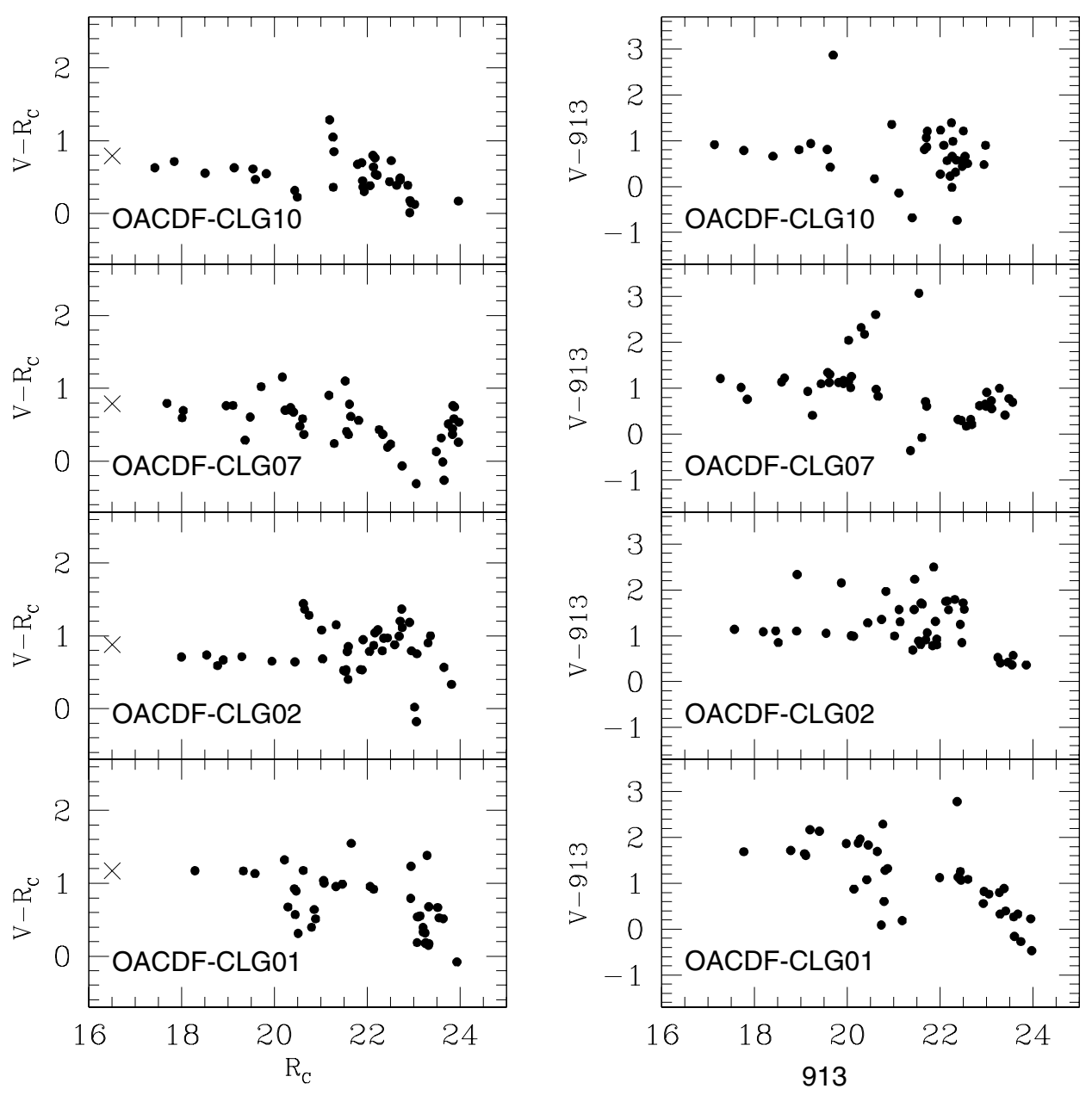

Fig. 10. Color-magnitude diagrams of our four best candidates, after applying the statistical subtraction of the background objects. The " $\times$ " symbol on the $\left(V-R_{\mathrm{C}}\right)$ vs. $R_{\mathrm{C}}$ diagrams represents the color corresponding to the photometric redshift reported in Table 5.

Table 5. Candidates for clusters of galaxies.

\begin{tabular}{lllllllllll}
\hline \hline Candidate & RA(2000) & Dec (2000) & richness & $\begin{array}{c}\text { iso-area } \\
\text { (pixs) }\end{array}$ & $S / N$ & ph_z \\
\hline OACDF-CLG01 & 12 & 23 & 58 & -12 & 44 & 53 & 59 & 30 & 5.7 & 0.3 \\
OACDF-CLG02* & 12 & 24 & 47 & -12 & 48 & 56 & 49 & 20 & 5.3 & 0.2 \\
OACDF-CLG03 & 12 & 23 & 56 & -12 & 27 & 29 & 36 & 26 & 4.3 & 0.5 \\
OACDF-CLG04 & 12 & 25 & 00 & -12 & 34 & 32 & 22 & 12 & 3.4 & 0.3 \\
OACDF-CLG05 & 12 & 25 & 27 & -12 & 46 & 14 & 25 & 17 & 3.7 & 0.3 \\
OACDF-CLG06 & 12 & 23 & 32 & -12 & 53 & 20 & 32 & 23 & 4.1 & 0.3 \\
OACDF-CLG07 & 12 & 25 & 04 & -13 & 01 & 41 & 65 & 32 & 6.1 & 0.2 \\
OACDF-CLG08 & 12 & 24 & 25 & -13 & 01 & 47 & 28 & 21 & 3.9 & 0.3 \\
OACDF-CLG09 & 12 & 23 & 18 & -13 & 02 & 58 & 17 & 8 & 3.3 & 0.1 \\
OACDF-CLG10 & 12 & 23 & 21 & -13 & 05 & 27 & 46 & 20 & 5.2 & 0.2 \\
\hline
\end{tabular}

*: This cluster has been spectroscopically confirmed.

cluster is also known as LCDCS 0522, and was first discovered in the Las Campanas Distant Cluster Survey (Gonzalez et al. 2001), which estimated the redshift to be $z \simeq 0.34$. Given the uncertainty of about 0.1 in the latter redshift, and considering that the redshift of this cluster was confirmed spectroscopically by us using spectra of seven galaxies in the cluster, we take as correct our redshift determination.

\section{Summary}

The OACDF, which comprises a photometric and astrometric database for galactic and extragalactic research, has been presented. The observations (imaging and spectroscopy), data reduction and photometric calibration, as well as the source extraction techniques, were described. 
During the course of the observations with the WFI at the ESO $2.2 \mathrm{~m}$ telescope, a photometric inhomogeneity was found that may be attributed, at least in part, to an additional-light pattern due to internal reflections off the telescope corrector, that may yield photometric errors of up to $3 \%$ in the $B, V, R$ and $I$ broad-bands and up to $5 \%$ in the medium-band filters.

The quality assessment studies of the OACDF provide the following results: the galaxy number counts of the OACDF deep data, in the $B, V$ and $R$ bands, are consistent with those from the literature; the resulting photometric redshifts, for objects brighter than about $V=23$, are consistent with the spectroscopic redshifts within a rms error of about 0.07 in the redshift range from 0 to 0.7 ; the distribution of photometric redshifts of OACDF sources is consistent with that of the CFRS at the $99.4 \%$ confidence level. The relationship between observed stellar colors and stellar spectral types for the 59 stars in our spectroscopic sample fits well the intrinsic color versus spectral type relation in the spectral range from A5 to M5. This confirms the low interstellar extinction of these stars, and hence the low galactic extinction in the OACDF.

We therefore conclude that the depth of the OACDF and its relatively large spatial coverage with respect to pencil beam surveys make it a good tool for extragalactic and stellar studies. In particular it is well suited for the definition of a statistically significant sample of early type galaxies for subsequent studies of galaxy formation and evolution in the redshift range $0-1$.

The OACDF also comprises a suitable database for the search for groups of galaxies and intermediate richness $\left(N_{\text {gal }}<\right.$ $100)$ clusters at intermediate redshift. Applying our cluster detection algorithms, several candidates for clusters of galaxies were detected above the $3 \sigma$ level. Using the criterion of the red sequence in the color-magnitude diagram, we selected 10 candidates with estimated richness in the range from 20 to 60 and a photometric redshift in the range from 0.2 to 0.5 . One of these (OACDF-CLG02) has been spectroscopically confirmed to have a redshift of 0.2 . The other 9 need spectroscopic confirmation. Future studies of these cluster candidates will allow us to improve our knowledge of the different evolutionary histories of galaxies in different enviroments. The multiband and sky coverage of the OACDF make it a suitable survey to test the predictions of different theoretical scenarios at intermediate and high redshift.

Acknowledgements. We thank the anonymous referee for comments and suggetions which helped to improve a first version of this paper. We also thank S. Andreon, G. Busarello, E. Covino, D. de Martino, M. Massarotti for discussions. The assistantship of G. Thereau during the first observing run contributed to make those nights more profitable. We are grateful to $\mathrm{H}$. McCracken for discussions regarding a first recipe for the astrometry. We thank Prof. M. Dopita for comments and suggestions on an earlier version of this paper. We also thank E. Bertin for many useful discussions and suggestions regarding cata$\log$ extraction methods and F. Valdes for several suggestions on the use of the IRAF mscred package. We are grateful to A. Di Dato, M. Colandrea and K. Reardon for their help with the INAF-OAC computers. Finally, we want to thank the ESO $2.2 \mathrm{~m}$ telescope team for their assistance during the observations. This project has been partially financed by the Italian Ministero dell'Università e della Ricerca Scientifica e Tecnologica (MURST).

\section{Appendix A: The catalog}

In this appendix we provide a catalog containing magnitudes and spectroscopic information for the 173 objects. The catalog is given in Tables A.1, A.2 and A.3.

The broad-band $A B$ magnitudes reported in the catalog can be transformed into the Johnson-Cousins system by applying the following expressions:

$$
\begin{aligned}
& V=0.870 \cdot V_{A B}+0.130 \cdot R_{A B}-0.030 \\
& R_{C}=1.030 \cdot R_{A B}-0.030 \cdot V_{A B}-0.221 \\
& I_{C}=0.907 \cdot I_{A B}+0.093 \cdot V_{A B}-0.462 \\
& B=1.311 \cdot B_{A B}-0.311 \cdot V+0.122
\end{aligned}
$$

\section{References}

Abraham, R. G., Glazebrook, k., McCarthy, P. J., et al. 2004 [arXiv:astro-ph/0402436]

Alcalá, J. M., Radovich, M., Silvotti, R., et al. 2002, in Survey and Other Telescope Technologies and Discoveries, ed. J. A. Tyson, \& S. Wolff, Proc. SPIE, 4836, 406

Alcock, C., Allsman, R. A., Alves, D. R., et al. 2000, ApJ, 542, 281

Arnaboldi, M., Aguerri, J. A. L., Napolitano, N. R., et al. 2002, AJ, 123,760

Aragon-Salamanca, A., Baugh, C. M., \& Kauffmann, G. 1998, MNRAS, 297, 427

Arnouts, S., Vandame, B., Benoist, C., et al. 2001, A\&A, 379, 740

Baade, D., Meisenheimer, K., Iwert, O., et al. 1998, The ESO Messenger, 93, 13

Bertin, E., \& Arnouts, S. 1996, A\&AS, 117, 393

Bolzonella, M., Miralles, J.-M., \& Pelló, R. 2000, A\&A, 363, 476

Bower, R. G., Lucey, J. R., \& Ellis, R. S. 1992, MNRAS, 254, 589

Brewer, J., \& Agustein, Th. 2000, WFI at MGP/ESO 2.2-m, Notes for observers

Bruzual, G., \& Charlot, S. 1993, ApJ, 405, 538

Burstein, D., \& Heiles, C. 1982, AJ, 87, 1165

Cimatti, A., Mignoli, M., Daddi, E., et al. 2002, A\&A, 392, 395

Cole, S., Lacey, C., Baugh, C. M., \& Frenk, C. S. 2000, MNRAS, 319 , 168

Colless, M., Dalton, G., Maddox, S., et al. 2001, MNRAS, 328, 1039

de Carvalho, R. R., \& Djorgovski, S. G. 1995, A\&AS, 187, 5302

Drory, N., Feulner, G., Bender, R., et al. 2001, MNRAS, 325, 550

Djorgovski, S. G., Gal, R. R., de Carvalho, R. R., et al. 2002, A\&AS, Meeting, BAAS, 34, 743

Ellis, R. H., Colless, M., Broadhurst, T., Heyl, J., \& Glazebrook, K. 1996, MNRAS, 280, 235

Faber, S. M., Friel, E. D., Burstein, D., \& Gaskell, D. M. 1985, ApJS, 57,711

Ferguson, H. C., Dickinson, M., \& Williams, R. 2000, ARA\&A, 38, 667

Fontaine, G., Brassard, P., \& Bergeron, P. 2001, PASP, 113, 409

Gladders, M. D., \& Yee, H. K. C. 2000, AJ, 120, 2148

Gonzalez, A. H., Zaritsky, D., Dalcanton, J. J., \& Nelson, A. 2001, ApJS, 137, 117

Heidt, J., Appenzeller, I., Gabasch, A., et al. 2003, A\&A, 398, 49

Huang, J.-S., Thompson, D., Kümmel, M. W., et al. 2001, A\&A, 368, 787

Iovino, A., Tassi, E., Mendes de Oliveira, C., Hickson, P., \& MacGillivray, H. 1999, IAUS, 186, 412

Iovino, A., de Carvalho, R. R., Gal, R. R., et al. 2003, AJ, 125, 1660 
Jacoby, G. H., Quigley, R. J., \& Africano, J. L. 1987, PASP, 99, 672

Jacoby, G. H., Hunter, D. A, \& Christian, C. A. 1984, ApJS, 56, 257

Jarrett, T.-H., Chester, T., Cutri, R., et al. 2000, AJ, 120, 298

Kauffmann, G., \& Charlot, S. 1998, MNRAS, 297, 23

Kormendy, J. 1977, ApJ, 218, 333

Kuemmel, M. W., \& Wagner, S. J. 2001, A\&A, 370, 384

La Barbera, F., Busarello, G., Merluzzi, P., Massarotti, M., \& Capaccioli, M. 2003, ApJ, 595, 127

Landolt, A. U. 1992, AJ, 104, 340

Le Fevre, O., Hudon, D., Lilly, S. J., et al. 1996, ApJ, 461, 534

Le Fevre, O., Mellier, Y., Mc Cracken, H. J., et al. 2003 [arXiv: astro-ph/0306252]

Lejeune, Th., Cuisinier, F., \& Buser, R. 1997, A\&AS, 125, 229

Lilly, S. J., Le Fevre, O., Crampton, D., Hammer, F., \& Tresse, L. 1995, ApJ, 455, 50 (catalog extracted from http:// wWw .astro.utoronto.ca/lilly/CFRS/CFRS_catalogue)

Maihara, T., Iwamuro, F., Tanabe, H., et al. 2001, PASJ, 53, 25

Manfroid, J., \& Selman, F. 2001, The ESO Messenger, 104, 16

Mc Cracken, H. J., Radovich, M., Bertin, E., et al. 2003, A\&A, 410, 17

Menanteau, F., Ellis, R. S., Abraham, R. G., Barger, A. J., \& Cowie, L. 1999, MNRAS, 309,208

Metcalfe, N., Shanks, T., Campos, A., McCracken, H. J., \& Fong, R. 2001, MNRAS, 323, 795
Monelli, M., Pulone, L., Corsi, C. E., et al. 2003, AJ, 126, 218

Navarro, J. F., Frenk, C. S., \& White, S. D. M. 1996, ApJ, 462, 563

Peebles, P. J. E. 2002, in A New Era in Cosmology, ed. N. Metcalfe, \& T. Shanks, ASP Conf. Ser., in press [arXiv: astro-ph/0201015]

Pickles, A. J. 1998, PASP, 110, 863

Puddu, E., Andreon, S., Longo, G., et al. 2001, A\&A, 379, 426

Puddu, E., De Filippis, E., Longo, G., Andreon, S., \& Gal, R. R. 2003, A\&A, 403, 73

Radovich, M., Arnaboldi, M., Ripepi, V., et al. 2004, A\&A, 417, 51

Somerville, R. S., Moustakas, L. A., \& Mobasher, B. 2004, ApJ, 600, 135

Stetson, P. 2000, Stetson Photometric Standards, web site: http://cadcwww.hia.nrc.ca/standards/

Thomas, D., Maraston, C., \& Bender, R. 2003, MNRAS, 339, 897

Turner, E. L., \& Gott, J. R. III 1976, ApJS, 32, 409

Tyson, J. A. 1988, AJ, 96, 1

White, S. D. M., \& Frenk, C. S. 1991, ApJ, 379, 52

Worthey, G., Faber, S. M., \& González, J. J. 1992, ApJ, 398, 69

Wolf, C., Meisenheimer, K., Rix, H.-W., et al. 2003, A\&A, 401, 73

York, D. G., Adelman, J., Anderson, J. E. Jr., et al. 2000, AJ, 120, 1579

Ziegler, B., Saglia, R., Bender, R., et al. 1999, A\&A, 346, 13 